Revue d'histoire de l'Amérique française

REVUE D.HISTOIRE DE L'AMÉRIQUE FRANÇAISE

\title{
Louis-Alexandre Taschereau et la législation sociale au Québec, 1920-1936
}

Antonin Dupont

Volume 26, numéro 3, décembre 1972

URI : https://id.erudit.org/iderudit/303194ar

DOI : https://doi.org/10.7202/303194ar

Aller au sommaire du numéro

Éditeur(s)

Institut d'histoire de l'Amérique française

ISSN

0035-2357 (imprimé)

1492-1383 (numérique)

Découvrir la revue

Citer cet article

Dupont, A. (1972). Louis-Alexandre Taschereau et la législation sociale au Québec, 1920-1936. Revue d'histoire de l'Amérique française, 26(3), 397-426. https://doi.org/10.7202/303194ar d'utilisation que vous pouvez consulter en ligne.

https://apropos.erudit.org/fr/usagers/politique-dutilisation/ 


\title{
LOUIS-ALEXANDRE TASCHEREAU ET LA LÉGISLATION SOCIALE AU QUÉBEC, 1920-1936 *
}

\author{
Antonin Dupont \\ Service des études \\ Commission des Ecoles catholiques \\ de Montréal
}

Louis-Alexandre Taschereau accède au poste de premier ministre du Québec dans la période de l'après-guerre, plus précisément en juillet 1920. Au cours des années précédentes, la solution à plusieurs problèmes avait été différée à cause de l'urgence de la situation. On situe aux environs de 1915 le moment où la population urbaine l'emporte sur la population rurale même s'il faut attendre le recensement de 1921 pour en obtenir la confirmation. En même temps que les villes voient augmenter le chiffre de leur population, plusieurs industries nouvelles s'implantent au Québec. Si la guerre n'a pas donné naissance à ces phénomènes, elle les a certainement accélérés. Ces nouvelles données sociales augmentent l'acuité des problèmes et exigent du gouvernement des mesures hardies.

A cinquante-deux ans, L.-A. Taschereau est investi de la plus haute autorité politique au Québec. Il n'en est pas cependant à ses premières armes comme homme politique puisqu'il compte déjà près de vingt ans de vie parlementaire. Elu député libéral de la circonscription de Montmorency en 1900, il est nommé ministre des Travaux publics en 1907 et devient l'un des principaux lieutenants de Lomer Gouin alors premier ministre du Québec. Le 26 août 1919, on lui confie le poste de procureur général ${ }^{1}$ qu'il conserve jusqu'à la fin de son mandat de premier ministre.

Plusieurs lois d'une portée sociale considérable ont été votées par le gouvernement Taschereau. Quelques-unes seulement feront l'objet d'un examen plus approfondi, soit qu'elles aient

* Une thèse intitulée: Les relations entre l'Eglise et l'Etat sous LouisAlexandre Taschereau, 1920-1936, a été soutenue avec succès par l'auteur, à l'Université McGill, en 1971. Afin d'obtenir un tableau plus complet du gouvernement Taschereau, le lecteur pourra s'y reporter.

1 Le terme juste est sans doute celui de ministre de la Justice. Cependant, tout au long de ce travail, et dans d'autres cas similaires, la terminologie employée sera celle qui est en usage à l'époque.

RHAF, vol. 26, no 3 (décembre 1972)

[ 397 ] 
mis à plus rude épreuve les relations entre l'Eglise et l'Etat, soit que leur incidence morale ait été plus marquée. La pauvreté de la documentation a influencé aussi le choix des mesures sociales à analyser. Les archives demeurent encore muettes sur la genèse de certaines lois et seul le temps permettra de découvrir les documents qui en expliquent le cheminement.

\section{Régie des alcools}

La première loi d'importance votée par le gouvernement Taschereau, et à laquelle il lie le sort de son administration, est la création d'une régie des alcools. Le Québec n'avait jamais été prohibitionniste. Malgré le vote de deux lois prohibitionnistes ${ }^{2}-$ la loi Dunkin, en 1864, par l'Union législative et la loi Scott, en 1878, par le gouvernement fédéral - les Québécois n'avaient pas abandonné la consommation de l'alcool. A l'occasion du référendum imposé au gouvernement Laurier, en 1898, par les tenants de la prohibition, le Québec s'était prononcé, par une forte majorité, contre l'adoption d'une loi prohibant l'importation, la fabrication ou la vente de l'alcool. Dans toutes les autres provinces du Canada, la majorité des citoyens avait voté en faveur de la prohibition. La campagne prohibitionniste menée par plusieurs associations nationales avait cependant rendu la population québécoise plus consciente des abus que pouvait provoquer l'usage de l'alcool. Dès le début du XXe siècle, l'Episcopat du Québec avait entrepris une grande croisade de tempérance. Mgr Paul Bruchési, archevêque de Montréal, et le cardinal Louis-Nazaire Bégin, archevêque de Québec, unirent leurs efforts et prêchèrent les bienfaits de la sobriété. Le zèle des autorités religieuses aiguillonna les tenants de la prohibition. Ils intervinrent auprès du gouvernement du Québec et obtinrent, en 1912, la création d'une commission d'enquête qui remet son rapport l'année suivante. Les commissaires ne recommandaient pas la suppression totale des boissons alcooliques mais ils proposaient plutôt une série d'amendements destinés à restreindre le commerce de l'alcool. ${ }^{3}$

Le déclenchement de la première Grande Guerre eut des répercussions sur les habitudes de vie des Américains. Les EtatsUnis, obligés d'alimenter l'Europe en produits agricoles, devaient

2 Consulter l'étude de Jean-Paul Lawlor, Le Commerce de l'alcool et la création de la Commission des liqueurs en 1921. Thèse de D.E.S. (Université de Montréal, 1970). Le mémoire de Jean-Paul Lawlor est fort bien documenté. Il s'intéresse surtout à l'aspect économique et commente les raisons qui peuvent expliquer l'intervention d'un gouvernement qui s'était fait l'ardent défenseur de l'entreprise privée. Cette étude représente, à notre avis, la meilleure recherche sur le sujet.

3 Le lecteur pourra consulter le Rapport de la Commission d'enquête sur le commerce des boissons alcooliques (Québec, février 1971). 
s'imposer des restrictions. D'une part, la nécessité de réserver l'orge et le blé au ravitaillement des troupes et des populations civiles et, d'autre part, le zèle inlassable des ligues prohibitionnistes ont forcé le gouvernement américain à interdire la fabrication, la vente ou le transport de l'alcool sur tout le territoire soumis à sa juridiction. La plupart des Etats avaient déjà adopté une législation semblable. En 1920, la loi Volstead était en vigueur à travers tout le pays.

Obéissant aux mêmes impératifs, le Canada décide, à l'automne de 1917, de défendre la fabrication et l'importation de toute boisson alcoolique. Huit des neuf provinces canadiennes sont assujetties à la loi; le Québec refuse obstinément de se laisser entraîner vers l'abstinence totale. A l'occasion d'un référendum organisé par le gouvernement québécois, la population se prononce en faveur de la prohibition mitigée. ${ }^{4}$ Le 1er mai 1919, la nouvelle loi entre en vigueur. ${ }^{5}$ Cependant, la mesure législative présentée par le trésorier Walter Mitchell devait donner lieu à de nombreux abus, ainsi qu'en témoigne J. Castell Hopkins :

Efforts along the line of moderate legislation had been made, in 1919 , the sale of whiskey prohibited except for special purposes, with the sale of beers and light wines, of specified alcoholic contents, permitted in cafés, etc. Abuses, however, developed and the law was not properly observed especially in Montreal. ${ }^{6}$

La rigueur de la loi est l'une des raisons qui expliquent les nombreux délits. Parmi les motifs corollaires, on peut signaler l'appât du gain et l'opposition de la majorité de la population à la prohibition. Plus les ventes d'alcool étaient nombreuses, plus les détenteurs de permis pouvaient arrondir leurs profits. Par ailleurs, les citoyens du Québec non seulement manifestaient peu de zèle dans l'observance de la loi mais, au contraire, s'employaient à découvrir les meilleurs moyens de la contourner.

Pour faire échec à la fraude et à la prévarication, le gouvernement québécois, bien loin de rendre la loi plus draconienne, propose, à la surprise générale, une mesure législative tout à fait nouvelle et s'engage, sur son honneur, à la faire observer. "Quel que soit le système auquel nous nous arrêtions et que doive voter la législature, nous verrons à ce que cette loi soit respectée."

4 Le Canada, 11 avril 1919.

5 Statuts de Québec, 9 George V (1919), chap. 19:100.

6 J. Castell Hopkins, The Canadian Annual Review of Public Affairs (Toronto, 1922), 660 .

7 Le Canada, 25 novembre 1920. 
Puisque, de l'avis d'un bon nombre, il se vend autant de boissons enivrantes sous le régime instauré par la loi de 1919 que sous un régime de vente libre, il vaut mieux libéraliser la consommation de l'alcool. Mais, afin d'éviter que le goût du lucre s'empare du vendeur, l'Etat devient le seul intermédiaire entre le fabricant et le consommateur. La loi créant la Commission des liqueurs est sanctionnée le 25 février 1921.8 Elle établit une régie composée de cinq membres et lui confie l'entière responsabilité de la vente de l'alcool. Seule la régie peut importer des pays étrangers et acheter directement des distilleries canadiennes les boissons alcooliques qu'elle revend ensuite au public dans des magasins qui sont sa propriété. Elle peut accorder des permis aux restaurants, hôtels, clubs, gares, etc., pour la vente des vins pendant les repas dans la salle à manger. Aux épiceries, elle peut permettre la vente de la bière seulement. Le vin et la bière pourront contenir une plus forte proportion d'alcool, soit respectivement 20 p.c. et 5 p.c. Une taxe de 5 p.c. est exigée des brasseries sur le produit brut de leurs ventes. ${ }^{9}$ La location et l'achat de terrains et d'édifices, le pouvoir de mener à terme toute transaction financière font partie des fonctions de la régie. Mais elle est aussi habilitée à exercer un rôle de surveillance et à poursuivre devant les tribunaux tous les violateurs de la nouvelle loi. Quant aux recettes que le Québec pourra tirer de la vente de l'alcool, elles font partie du fonds consolidé du revenu de la province. La régie des alcools rend compte au trésorier du Québec de toutes ses activités. Un représentant du Conseil des ministres peut être nommé pour agir comme vérificateur auprès de la régie d'Etat. ${ }^{10}$

Par cette loi, Louis-Alexandre Taschereau étatise la vente de l'alcool et du vin. En posant ce geste, le gouvernement se substitue à l'entreprise privée. Mais ce qui surprend davantage, c'est que partout ailleurs en Amérique les populations vivent sous le régime de la prohibition. Le Québec est le seul Etat, au nord du Rio Grande, où la vente de l'alcool est tolérée. Il faut donc reconnaître l'audace du gouvernement québécois qui s'attaque à un problème que l'on considérait jusque-là insoluble. A l'avenir, tout citoyen du Québec pourra se procurer une bouteille d'alcool sans avoir à en prouver la nécessité par une ordonnance médicale et sans être obligé de s'adresser à ceux qui le fabriquent dans la clandestinité.

L'initiative du gouvernement est diversement appréciée selon les milieux. Jules Picard emploie le ton sarcastique:

${ }^{8}$ Statuts de Québec, 11 George V (1921), chap. 24: 69.

9 Jean-Paul Lawlor, op. cit., 72.

10 Ibid., 74. 
Le gouvernement n'est pas étatiste, mais le premier en Amérique il s'improvise mastroquet, le premier, il fonde un monopole d'Etat des spiritueux. Il n'est pas étatiste; il est un précurseur, un novateur en étatisme.11

Mise en vigueur le 1er mai 1921, la loi est âprement défendue par la presse libérale. "En prenant le contrôle des alcools, le gouvernement Taschereau a non seulement adopté une mesure de tempérance, il a encore assuré à la province une source considérable de revenus, comme l'avenir le prouvera. ${ }^{12}$ " Dès l'année suivante, cette opinion est confirmée. Le rapport officiel de la régie des alcools déclare un surplus de quatre millions de dollars. A la session de 1929 , un million de dollars provenant des profits de la régie des alcools est versé aux œuvres d'assistance publique. ${ }^{13}$

Force nous est de reconnaître que la loi votée par le gouvernement Taschereau place le Québec à l'avant-garde des Etats de l'Amérique du Nord. Aussi, ne faut-il pas s'étonner de ce que la création d'une régie des alcools soulève tant d'opposition. Au parti conservateur et aux nationalistes s'allient une large fraction du clergé et les principaux organes de la presse catholique dans une campagne de protestations contre la réforme mise en vigueur par l'administration libérale. En dépit des nombreux reproches que lui vaut la défense opiniâtre de la loi des alcools, Louis-Alexandre Taschereau refuse de chanter la palinodie. $\mathrm{Ni}$ Henri Bourassa, ni l'abbé Edouard-V. Lavergne ne réussissent à le faire fléchir. Cependant, à chacune des sessions de l'Assemblée législative, le gouvernement présente des amendements en vue d'améliorer la loi.

Durant les seize années de son mandat de premier ministre, L.-A. Taschereau subit les assauts des prohibitionnistes. La réponse vient des provinces du Canada qui, tour à tour, se rangent dans le camp du Québec. Mais l'appui le plus éclatant est assuré par les Etats-Unis qui, en décembre 1933, votent le 21 $1^{\mathrm{e}}$ amendement à la Constitution et abolissent la loi Volstead. A l'avenir, les Américains pourront consommer librement de l'alcool. Cependant, à la différence du Québec, le commerce des boissons alcooliques demeure entre les mains de l'entreprise privée.

L'Episcopat du Québec, comme dépositaire de l'autorité religieuse, n'a jamais dénoncé la loi. Quelques représentants de la hiérarchie catholique de la province n'ont guère nourri de sentiments très bienveillants envers la régie des alcools du premier

11 Jules Picard, Le Nationaliste, 20 mars 1921.

12 Le Canada, 19 décembre 1921.

13 Le Canada, 7 février 1929. 
ministre Louis-Alexandre Taschereau. La presse catholique a manifesté ouvertement et nettement son hostilité au projet de loi d'abord, à la loi ensuite, puis faisant contre mauvaise fortune bon cœur, a accepté le principe tout en se plaignant amèrement de son application. Parmi les catholiques du Québec, membres du clergé ou simples fidèles, plusieurs ont pris parti contre la loi, en sont demeurés des adversaires irréductibles et n'ont pas ménagé leurs efforts pour que la prohibition subsiste, au moins localement.

\section{Assistance publique}

La réaction négative des milieux catholiques à l'étatisation de la vente de l'alcool va se muer en une opposition virulente contre l'intervention de l'Etat dans le champ du bien-être social. L'urbanisation avait engendré de nombreux problèmes et la charité privée était impuissante à les résoudre. Mais en voulant participer aux œuvres de prévoyance sociale, l'Etat pénètre dans un domaine jusque-là réservé à l'Eglise et rompt ainsi avec une vieille tradition.

Si l'on examine les lois en vigueur sur le continent américain relativement à l'assistance publique, il faut convenir que le Québec n'avait pas fait preuve de beaucoup de dynamisme. Le Manitoba, en 1916, avait adopté la loi intitulée Mothers' Allowance Act; la Saskatchewan et l'Alberta avaient mis sur pied, en 1917, un service municipal d'hospitalisation gratuite. Au NouveauBrunswick et en Nouvelle-Ecosse, la Poor Law remettait le fardeau de l'assistance à la municipalité. ${ }^{14}$ L'Ontario et les EtatsUnis firent appel à des services publics d'assistance, les Charity Organisation Societies dont les deux grands objectifs étaient de supprimer le paupérisme et de sauver la cité industrielle.

Malgré le retard du gouvernement québécois à pénétrer dans le champ de l'assistance sociale, la réaction n'en est pas moins fort violente. Les critiques les plus virulentes s'expriment en dehors de l'Assemblée législative. C'est le cinquième article de la loi ${ }^{15}$ qui soulève l'ire de la presque totalité des opposants. Il confère à l'Etat le droit non seulement d'accorder des subven-

${ }^{14} \mathrm{~A}$ la fin de 1919 , le déficit des institutions de bienfaisance s'élève à plus d'un quart de million de dollars. C'est pourquoi, au début de 1920, Québec se voit dans l'obligation de sanctionner une mesure qui accorde aux institutions le pouvoir de refuser l'assistance aux personnes dans le besoin si la municipalité qu'ils habitent depuis six mois ne paie pas le coût de leur hébergement. Les municipalités ne pouvant satisfaire à cette exigence, l'application de la loi devient impossible. Le gouvernement doit donc trouver une autre solution au problème de l'assistance.

15 Annuaire statistique du Québec, 8 (Québec, 1921) : 222. 
tions aux institutions reconnues d'assistance publique mais l'autorise à en surveiller l'utilisation. Jusqu'alors, le gouvernement avait accordé des subventions très modiques à différentes institutions sans que celles-ci ne soient soumises à aucune surveillance. Aussi, dès que l'Etat manifeste sa volonté de majorer les subventions et, en même temps, de soumettre les maisons d'assistance à une réglementation précise, il encourt aussitôt les foudres de nombreux opposants. Henri Bourassa est l'un des adversaires les plus irréductibles de la loi:

Je ne méconnais ni les vices ni les dangers du système; mais dans le cas des 165 institutions de bienfaisance auxquelles M. Taschereau vante les beautés du mariage mixte et polygamique, le danger serait considérablement aggravé: le chef virtuel de la communauté serait l'Etat, son autorité maritale s'exercerait sur toutes les activités de ses multiples épouses, les institutions subventionnées; et, par l'enchaînement des causes et des effets, elle ne tarderait pas à pénétrer jusque dans la vie intérieure des congrégations religieuses vouées aux œuvres de bienfaisance. Le cas, on le voit, est beaucoup plus complexe. ${ }^{18}$

La loi de l'Assistance publique ${ }^{17}$ est l'occasion d'une démarche dont on a peu d'exemples dans l'histoire du Québec. Les élites cléricale et politique peuvent encore s'affronter et on ne peut prétendre que les deux pouvoirs ont les mêmes vues sur tous les problèmes. Les querelles politico-religieuses n'ont pas cessé avec la fin du XIX ${ }^{\mathrm{e}}$ siècle. ${ }^{18}$ Le 11 janvier 1922 , tous les évêques du Québec adressent une lettre confidentielle au premier ministre Louis-Alexandre Taschereau. En plus d'exprimer ses vues sur

16 Henri Bourassa, Une mauvaise loi (Montréal, 1921), 21-22.

17 Dans l'annexe 2 du Rapport de la Commission royale d'Enquête sur les problèmes constitutionnels, l'étude de Gonzalve Poulin, intitulée "L'Assistance sociale dans la province de Québec, 1608-1951", explique abondamment l'évolution de cette question sociale. Le lecteur pourra se reporter à ce document s'il veut mieux comprendre nos commentaires.

A consulter, la thèse de maîtrise de Pauline Godbout, intitulée Etude de la législation sociale de la province de Québec (1921-1939) (Faculté des Sciences sociales, Université Laval, 1954). L'absence de références précises et le peu de diversité des sources enlèvent une certaine valeur à l'étude. Egalement, des affirmations gagneraient à être nuancées.

A consulter également, le Rapport du Comité d'étude sur l'assistance publique (Québec, 1963). - Serge Mongeau, Evolution de l'assistance au Québec (Montréal, 1967).

18 Dans l'Histoire des Canadas, les: auteurs (Rosario Bilodeau, Robert Comeau, André Gosselin et Denise Julien) laissent entendre que le pouvoir clérical ne sera plus contesté après 1896 . Or, la plupart des ouvrages cités dans la note précédente soulignent que l'on revient à l'année 1885 et qu'on rejoint l'opposition contre les asiles. On dit même que la législation sociale de l'époque a été fortement influencée par la loi de l'Assistance publique. 
l'immunité des biens ecclésiastiques et sur le suffrage féminin, l'Episcopat formule la supplique suivante:

Notre troisième vœu concerne la loi de l'assistance publique votée l'an dernier, nous en sommes sûrs, avec les meilleures intentions du monde, mais qui n'est pas sans provoquer de vifs commentaires et sans produire de très graves appréhensions.

La charité privée, dans notre province, a fait et continue de faire chaque jour des merveilles. Avec des ressources très limitées, mais dont l'économie et le dévouement ont su tirer un admirable parti, nos institutions catholiques de bienfaisance ont accompli des œuvres de toutes sortes, adaptées à tous les besoins, et que l'on ne saurait assez louer. Ce gouvernement ne pourrait-il pas venir en aide à ces institutions dont les charges vont croissant, sans leur imposer les formalités très gênantes de la loi de 1921 ? Pourquoi ne pas remplacer cette loi de l'Assistance publique par une autre plus simple que nous pourrions agréer? Pourquoi, du moins, ne pas modifier la loi existante de telle sorte que l'on y ferait pleine confiance aux établissements catholiques de charité et l'on y tiendrait compte de la juste indépendance de ces institutions placées dans chaque diocèse sous le contrôle de l'évêque ? Une législation qui, tout en respectant les autres croyances, s'harmonise avec la croyance et la discipline catholique de la très grande majorité des habitants d'une Province, n'a rien qui puisse offusquer personne. ${ }^{19}$

En somme, les évêques du Québec veulent que les institutions catholiques ne soient pas assujetties à la loi. Quant aux institutions appartenant à d'autres minorités religieuses, ils laissent toute liberté au gouvernement. Mais la hiérarchie catholique préférerait que l'on conserve l'ancienne loi, tout en souhaitant une aide accrue. Le chef du gouvernement du Québec refuse d'obtempérer au désir exprimé par l'Episcopat et demeure intraitable sur cette question. Dans une réponse respectueuse mais ferme, il exprime clairement sa volonté de poursuivre l'application de la loi:

(...)

Je crois que le gouvernement a donné assez de preuves de son désir de coopérer avec les autorités religieuses pour que celles-ci se considèrent à l'abri des dangers que craint ce légiste. ${ }^{20}$ Il suffit de mentionner ce que nous avons fait en

${ }^{19}$ ANQ, Fonds Louis-Alexandre Taschereau, Lettre des archevêques et évêques du Québec à Louis-Alexandre Taschereau, 11 janvier 1922.

20 A la lettre de l'Episcopat est attaché un projet de loi d'assistance publique fait par un légiste. Malheureusement, nous n'avons pu retrouver ce dernier document. Le légiste serait vraisemblablement le notaire Joseph Sirois. 
faveur de nos universités catholiques, ce que nous faisons actuellement en faveur de nos collèges classiques, pour démontrer que nous ne méritons pas d'être tenus en suspicion. $(\ldots)$

Si l'on n'en veut pas, je suis prêt à demander à mes collègues de la révoquer, laissant la responsabilité des misères qui en résulteront aux critiques de cette loi.

(...)

Quand notre loi a été soumise aux autorités ecclésiastiques, l'année dernière, avant sa présentation à la Chambre, c'était le temps, il me semble, de nous dire qu'elle était païenne. Un des évêques, signataires de la lettre, m'a dit qu'il l'approuvait de tout cœur. ${ }^{21}$

(...)

A la session de janvier 1922, lorsque Arthur Sauvé, chef de l'Opposition, demande la production du document confidentiel des évêques, Louis-Alexandre Taschereau, après avoir communiqué avec Mgr Paul-Eugène Roy, représentant de l'Episcopat, lui déclare qu'il ne peut le rendre public, les évêques du Québec ne voulant pas "prendre ni les Chambres ni le public comme témoins ou interprètes de leur confidence". ${ }^{22}$

C'est ainsi que commence à poindre les premiers signes d'affrontement dans les relations entre l'Eglise et l'Etat sous LouisAlexandre Taschereau. Pour être plus subtile, la contestation n'en sera pas moins réelle. Entre les deux pouvoirs, quelques embellies viendront tempérer momentanément le climat de tension, mais il se trouvera toujours quelque événement ou quelque personnage pour soulever de nouveau des vents de tempête. La loi de l'Assistance publique est la première occasion sérieuse de conflit. Cependant, grâce aux bons offices de Mgr FrançoisXavier Ross, évêque de Gaspé, et après plus de trois ans d'attente, un premier amendement est ajouté à la loi, à la session de 1924:

Tout contrat fait à compter du (date de l'entrée en vigueur de la présente loi) avec une institution de charité dirigée par une communauté religieuse catholique romaine, doit recevoir l'approbation de l'Ordinaire du diocèse dans lequel est située la dite communauté. ${ }^{23}$

21 ANQ, Fonds Louis-Alexandre Taschereau, Lettre de Louis-Alexandre Taschereau à Mgr Paul-Eugène Roy, 20 janvier 1922.

22 ANQ, Fonds Louis-Alexandre Taschereau, Lettre de Mgr PaulEugène Roy à Louis-Alexandre Taschereau, 22 janvier 1922.

23 Statuts de Québec, 14 George V (1923-24), chap. 48: 164. 
Dans la livraison de juillet 1924 de l'Action française, ${ }^{24}$ l'un des collaborateurs de la revue s'en prend d'un ton persifleur, au premier ministre et à sa loi de l'Assistance publique. Le chef du gouvernement s'adresse à Mgr Georges Gauthier, archevêque coadjuteur de Montréal, et le met en demeure de désavouer les propos publiés dans la revue qui a pour directeur l'abbé Lionel Groulx, prêtre du diocèse de Montréal. Il souligne discrètement que l'hôpital des Incurables de Montréal a demandé une subvention et qu'il aimerait pouvoir la lui accorder..$^{25}$ Dans une première lettre, Mgr Gauthier signale que "la question de l'Assistance est devenue, par suite de circonstances dans lesquelles je ne suis pour rien, une question de politique militante, et je croirais tout à fait inopportun que l'un des miens prît sur ce point, avant que je l'en aie chargé, une attitude quelconque". ${ }^{26}$ En réponse à une deuxième lettre du premier ministre, le chef spirituel du diocèse de Montréal déclare qu'il ne peut donner son avis sur la loi de l'Assistance publique même au risque de compromettre l'œuvre des Incurables :
(...)
Je suppose bien que mon avis, si je le donnais, ne tarderait pas à être connu, et je me trouverais sans excuse si l'on me disait un jour que, dans l'expression d'une opinion, je me suis laissé guider par un autre motif que celui du devoir. Je veux écarter de pareils actes, jusqu'à l'ombre même d'un sentiment intéressé. Vous penserez comme moi que la con- fiance, l'estime et le respect de ceux qui relèvent de moi sont à ce prix. ${ }^{27}$

C'est en 1925 qu'est soumis l'amendement qui doit emporter l'adhésion des cinq évêques encore irréductibles. "Dans l'application de ces règlements, comme dans le fonctionnement de la présente loi, lorsqu'il s'agit de communautés religieuses catholiques, rien ne pourra préjudicier aux droits de l'évêque sur ces communautés, ni à leurs intérêts religieux, moraux et discipli-

24 A.V. [Anatole Vanier], "Le Québec et la France", L'Action française, 12 (juillet 1924) : 58.

25 ANQ, Fonds Louis-Alexandre Taschereau, Lettre de Louis-Alexandre Taschereau à Mgr Georges Gauthier, 15 août 1924 .

26 ANQ, Fonds Louis-Alexandre Taschereau, Lettre de Mgr Georges Gauthier à Louis-Alexandre Taschereau, 21 août 1924.

27 ANQ, Fonds Louis-Alexandre Taschereau, Lettre de Mgr Georges Gauthier à Louis-Alexandre Tachereau, 9 septembre 1924 . 
naires." ${ }^{28}$ Le 5 février 1929, Athanase David ${ }^{29}$ annonce qu'une somme de $\$ 1,000,000$ provenant des profits de la régie des alcools sera versée, chaque année, à l'Assistance publique. ${ }^{30}$

L'épisode de l'Hôtel-Dieu de Québec vient entretenir la division entre les autorités civiles et religieuses. Jusqu'ici, on avait pourvu au soin des malades sans l'aide du gouvernement. En août 1930, la communauté réclame une subvention de $\$ 400,000$ pour agrandir cet hôpital et en améliorer les services. A sœur Saint-Dominique, supérieure de l'Hôtel-Dieu, Louis-Alexandre Taschereau explique les conditions selon lesquelles la subvention peut être accordée. Si l'institution veut se soumettre à la loi de l'Assistance publique, le gouvernement est prêt à verser un montant de $\$ 300,000 .{ }^{31}$ Explicitement, cela signifie que le gouvernement accepte de verser une subvention à l'Hôtel-Dieu de Québec si ses dirigeants consentent à ouvrir leurs livres aux vérificateurs de l'Assistance publique. Les religieuses repoussent cette contrainte. A l'intervention personnelle du cardinal Raymond-Marie Rouleau, archevêque de Québec, Louis-Alexandre Taschereau répond par les mêmes arguments qu'il a utilisés auprès de la supérieure de l'Hôtel-Dieu. ${ }^{32}$ Il réitère à $\mathrm{Mgr}$ Omer Plante, évêque auxiliaire de Québec, son désir d'en arriver à un règlement. "Il est possible que nous puissions trouver une autre formule également acceptable, et je suis à la disposition de Votre Grandeur, et de l'Hôtel-Dieu, pour en discuter les termes, lorsque vous le désirerez." ${ }^{33}$ La dernière pièce à verser au dossier de l'Hôtel-Dieu est la lettre du premier ministre Taschereau, du 19

28 Statuts de Québec, 15 George V (1925), chap. 55: 167.

29 Athanase David, né en 1885, était le fils de Laurent-Olivier David. Nommé secrétaire provincial, le 25 août 1919 , il s'occupait plus particulièrement du domaine intellectuel et du domaine sanitaire. On lui doit entre autres choses: la réforme de l'enseignement primaire, secondaire, technique et supérieur; le vote d'un crédit annuel aux collèges classiques; la création de bourses permettant aux diplômés les mieux doués des universités d'aller étudier en Europe; l'établissement d'un prix littéraire annuel dit "prix David" et attribué par jury; la fondation des écoles de beaux-arts en 1922; la loi de l'Assistance publique en 1921. Il a été pendant plusieurs années député de Terrebonne, puis sénateur à Ottawa.

30 Le Canada, 7 février 1929. "Le trésorier de la province est autorisé à verser annuellement, une somme de un million de dollars, provenant des revenus nets des opérations de la Commission des Liqueurs de Québec, au Fonds de l'Assistance publique." (Statuts de Québec, 19 George V (1929), chap. $63: 189$.)

31 ANQ, Fonds Louis-Alexandre Taschereau, Lettre de L.-A. Taschereau à soeur Saint-Dominique, 12 août 1930 .

${ }_{32}$ ANQ, Fonds Louis-Alexandre Taschereau, Lettre de L.-A. Taschereau au cardinal Raymond-Marie Rouleau, 22 septembre 1930.

33 ANQ, Fonds Louis-Alexandre Taschereau, Lettre de Louis-Alexandre Taschereau à Mgr Omer Plante, 27 octobre 1930. 
février 1931, au cardinal R.-M. Rouleau. Après avoir rappelé la discussion qu'ils ont eue ces jours derniers au sujet de l'HôtelDieu, il mentionne qu'il est venu près de s'entendre avec sœur Saint-Dominique. Sous le même pli, il fait parvenir au Cardinal la copie de la lettre de sœur Sainte-Marguerite, supérieure de l'Hôtel-Dieu de Chicoutimi, qui remercie le premier ministre de l'aide financière que son institution a reçue. ${ }^{34}$ La mort du cardinal Rouleau, survenue le 30 mai, a rompu les négociations et la subvention de $\$ 300,000$ n'a pas été accordée.

\section{L'Adoption}

La loi de l'Adoption votée par le gouvernement du Québec en 1924 corrige une situation que l'on déplorait depuis fort longtemps. Aucun article du code civil ne protège les orphelins et les parents adoptifs. A la demande de communautés religieuses qui prennent soin des orphelins et de représentants du clergé, le procureur général dépose un projet de loi à l'Assemblée législative, le 27 février 1924. Henri Bourassa manifeste aussitôt son opposition:

Si cette législation extraordinaire entre dans le corps de nos lois, elle allongera la liste des atteintes portées à tout ce qui a fait la force et la vitalité de notre ordre social. Elles se sont singulièrement multipliées, depuis quelques années. Notre usine législative prend des allures inquiétantes. Que les hommes d'affaires ou d'intrigue, exclusivement préoccupés de soucis d'argent, ne s'en inquiètent pas, cela s'explique: là où est leur trésor, là est leur cœur. L'insouciance des hommes de principes est moins facile à comprendre. ${ }^{35}$

Cette critique est le premier coup de clairon d'une campagne de protestations. L'avocat Léo Pelland, l'abbé Antonio Huot et l'éditorialiste Jules Dorion attaquent tour à tour la loi de l'Adoption sanctionnée le 15 mars 1924. Ce qui étonne encore une fois, c'est l'obstination avec laquelle la presse catholique et quelques coryphées jansénistes dénigrent les gestes posés par le gouvernement. Dès que l'Etat intervient, on suspecte aussitôt ses intentions. Pourtant, il ne fait que se rendre aux objurgations des milieux catholiques. Il ne tente aucunement de brimer les intérêts de qui que ce soit. Tout en ne cachant pas leur surprise devant la réaction hostile d'une partie de la presse catholique et de certains membres du clergé, les autorités civiles sont prêtes à entreprendre de nouvelles négociations.

${ }^{34}$ ANQ, Fonds Louis-Alexandre Taschereau, Lettre de Louis-Alexandre Taschereau au cardinal R.-M. Rouleau, 19 février 1931.

35 Henri Bourassa, Le Devoir, 7 mars 1924. 
Dans les mois qui suivent la sanction de la première loi de l'Adoption, le gouvernement entame des pourparlers avec les autorités religieuses par l'intermédiaire de Mgr Laflamme, curé de la Basilique de Québec. A la fin de la session de 1925, le premier ministre propose des modifications. Relativement à l'ancienne loi, la nouvelle contient deux dispositions importantes:

1. l'adoption doit être faite par deux époux vivant légitimement ensemble;

2. l'adoptant, dans le cas d'une veuve ou d'un veuf, doit avoir vingt ans de plus que l'adopté. ${ }^{36}$

Il est une stipulation de la nouvelle loi qui en réjouit plusieurs, c'est celle de l'article 4 "décrétant que l'adoptant doit professer la même foi religieuse que celle à laquelle appartient l'adopté par le baptême". ${ }^{37}$ L'abbé Antonio Huot exprime sa satisfaction:

Nous sommes heureux de dire aujourd'hui que notre confiance n'a pas été trompée. Sur l'initiative de l'Hon. Alexandre Taschereau, en effet, la législature vient de voter à l'unanimité une refonte de la Loi de l'Adoption de 1924 faite dans le meilleur sens, après entente entre le gouvernement et $\mathrm{Mgr}$ Laflamme, curé de Québec, agissant pour le compte de l'autorité diocésaine. Il nous fait plaisir de féliciter sincèrement l'Hon. Premier Ministre et ses collègues de cette initiative des plus heureuse. ${ }^{38}$

Cette loi de l'Adoption est la première à apparaitre dans les statuts du Québec. Ce n'est qu'en 1924 qu'on substitue l'adoption légale à l'adoption de fait. Placés devant le problème de l'enfance malheureuse, les législateurs québécois "jugèrent à propos de doter la province d'une loi qui réglementerait l'adoption en mettant fin à la situation non juridique où elle s'était trouvée dans le passé". ${ }^{39}$ Par cette innovation, Louis-Alexandre Taschereau ajoutait au code civil du Québec promulgué le 1er août 1866. A ce titre, on doit considérer la loi de l'Adoption comme une réforme importante du gouvernement Taschereau. Une mesure législative prend de la valeur autant par les principes qu'elle fixe que par le nombre d'individus qui lui sont soumis.

${ }^{36}$ La Presse, 25 mars 1925. 1951), 85 .

37 Hervé Roch, L'Adoption dans la province de Québec (Montréal, 38 Antonio Huot, "Lois heureusement amendées", La Semaine religieuse de Québec, 37 (avril 1925): 486.

39 Hervé Roch, L'Adoption..., op. cit., 26. 


\section{Cinéma et observance du dimanche}

Les questions du cinéma et de l'observance du dimanche ont fortement contribué à tendre les relations entre l'Eglise et l'Etat. La popularité croissante du septième art soulève les appréhensions des autorités religieuses. Dès 1921, Mgr Paul Bruchési, dans une lettre pastorale, exprime sa désapprobation: "Chaque jour, dans les grandes et petites villes, des milliers et des milliers d'enfants, de jeunes gens et de jeunes filles, de femmes et d'hommes, pénètrent dans les salles obscures et malsaines." ${ }^{40}$ A l'époque, le Québec est le seul endroit au Canada où l'on peut aller au cinéma le dimanche. Au surplus, les enfants de moins de seize ans peuvent être admis au cinéma s'ils sont accompagnés de leurs parents ou d'une personne responsable. Voilà pourquoi, au cours d'un congrès de la Confédération des Travailleurs catholiques du Canada, tenu à Hull en septembre 1921, une résolution est adoptée demandant "aux autorités provinciales d'interdire par une loi ou règlement, l'entrée du cinéma aux enfants de moins de seize ans, qu'ils soient ou non accompagnés de leurs parents". ${ }^{41} \mathrm{Au}$ mépris de la loi et avec la connivence des propriétaires de cinémas, nombreux sont les enfants qui assistent à des représentations cinématographiques sans être accompagnés.

Un tragique événement va porter au premier plan de l'actualité la question du cinéma. Le dimanche 9 janvier 1927, un incendie éclate au cinéma Laurier Palace; la panique s'empare alors des spectateurs et soixante-dix-huit enfants meurent asphyxiés. Sous la pression des autorités religieuses et de diverses associations, le gouvernement institue une commission d'enquête. A la suite du rapport du juge Louis Boyer, ${ }^{42}$ seul commissaire enquêteur, le premier ministre, procureur général, présente une loi interdisant l'accès des cinémas aux enfants de moins de seize ans. On y ajoute toutefois une exception en faveur des collèges, des couvents ou des autres institutions d'enseignement qui pourront donner des représentations cinématographiques gratuitement sans avoir à tenir compte de l'âge. ${ }^{43}$

Les adversaires du cinéma dominical n'ont pas obtenu la fermeture des salles le dimanche. Malgré l'intervention des autorités diocésaines de Montréal et la présentation d'une requête

40 Mgr Paul Bruchési, "Les maux de l'heure présente", La Semaine religieuse de Montréal, 77 (avril 1921) : 247.

41 "Le cinéma et les enfants", L'Action française, 7 (février 1922) : 85.

42 Le Soleil, 31 août 1927. Le rapport a été remis au gouvernement le 30 août 1927.

43 Statuts de Québec, 18 George V (1928), chap. 60: 193. 
couverte de nombreuses signatures, le premier ministre du Québec considère le cinéma du dimanche comme une nécessité sociale et l'une des rares distractions que peuvent s'offrir les ouvriers. Aussi, respectant scrupuleusement les recommandations du rapport Boyer, il n'y interdit que la présence des enfants âgés de moins de seize ans. En même temps, il permet aux communautés religieuses et aux évêchés, propriétaires de couvents et de collèges, de recevoir des enfants au-dessous de seize ans. Or, la plupart des salles de ces institutions où se donnent des représentations cinématographiques n'ont pas été construites en fonction de ces divertissements. Malgré le danger qu'elles constituent, le gouvernement autorise cette dérogation sous prétexte que les spectacles y sont gratuits.

L'autorité civile peut difficilement interdire le cinéma dominical. Il est entré dans les mœurs et les tribunaux se sont prononcés contre une telle interdiction. La loi sanctionnée le 28 mars 1928 tient compte de l'état d'esprit de la population du Québec et, par une concession, tente de faire taire les opposants, en l'espèce, les autorités religieuses et quelques associations.

Intimement liée à l'observance du dimanche, la question du cinéma entraîne à parler du précepte dominical. Les progrès de l'industrie de la pulpe et du papier engendrent des bouleversements sociaux et le respect du dimanche est l'une des traditions catholiques qui a le plus à souffrir du nouvel état de choses. Par ailleurs, le travail représente un adversaire beaucoup plus redoutable que le cinéma dans la lutte de l'Eglise en faveur du précepte dominical. Nous retrouvons, à peu de chose près, les mêmes antagonistes en présence. D'un côté, les puissances financières, le gouvernement et les syndicats neutres; de l'autre, l'Eglise, les sociétés catholiques et la bonne presse. Les autorités religieuses, très conscientes de la menace, s'emploient à dénoncer les violateurs du dimanche:

Pourquoi faut-il, que dans bon nombre de centres industriels, nous ayons constamment sous les yeux l'attristant spectacle d'usines en pleine activité, une grande partie du jour consacré au Seigneur, et de nombreux ouvriers employés d'une façon continue à des travaux que rien ne justifie, mais que seul l'esprit de lucre peut expliquer. Car on ne peut le nier, dans beaucoup de nos manufactures, le travail de fabrication se prolonge régulièrement jusqu'à sept ou huit heures le dimanche matin. ${ }^{44}$

${ }^{44}$ Lettre pastorale du cardinal Bégin citée par L'Action catholique, 30 avril 1923. 
Le gouvernement est souvent pris à partie car, malgré ses bonnes dispositions, les violations n'en continuent pas moins; à tel point que les évêques ont cru bon de faire une dénonciation solennelle qui oblige le procureur général à "se mettre immédiatement à l'œuvre pour recueillir les preuves juridiques qui lui permettront d'assurer le respect du dimanche"..55

La tâche de l'autorité civile n'est pas de tout repos. Elle doit concilier les impératifs de la production industrielle avec les principes religieux de la population. Interdire tout travail le dimanche, c'est placer l'industrie dans une situation fort difficile; ne pas intervenir et faire la sourde oreille, c'est soulever l'ire d'associations comme la Ligue du Dimanche et mécontenter l'Episcopat. Aussi, ne doit-on pas se surprendre de voir le gouvernement adopter une solution de compromis. Celui-ci n'intervient qu'après plusieurs dénonciations ou lorsque la situation devient trop tendue. Le souci du premier ministre de ne pas perturber le développement industriel du Québec est fort peu apprécié des partisans du repos dominical:

Que les étrangers s'emparent de nos richesses, de nos forêts, de nos pouvoirs d'eau; qu'ils les exploitent et les épuisent; et qu'ils emploient nos gens pour scier leur bois, c'est inévitable, puisqu'ils ont les capitaux. Mais, au moins, qu'ils le fassent dans les mêmes conditions que nous. C'est une concurrence déloyale de leur part que de faire travailler nos gens le dimanche pour produire, eux, tous les jours de la semaine, alors que cela nous est interdit, à nous qui voulons respecter les lois du pays. Quand ils auront enlevé tout notre bois, ils s'en iront; mais ils nous laisseront le mal moral qu'ils auront introduit dans nos mœurs. ${ }^{46}$

La grande association nationale de l'industrie du papier tient son congrès à Montréal, le 28 janvier 1927. Les chefs d'entreprise font valoir auprès des représentants du gouvernement la nécessité pour eux de poursuivre, le dimanche, certaines activités industrielles. L'Episcopat, le clergé, la presse catholique et les syndicats nationaux protestent auprès du gouvernement pour qu'il interdise les travaux de réparation et d'entretien le dimanche. Donner satisfaction aux deux parties, c'est presque demander de dénouer le nœud gordien.

Si les petites villes industrielles sont davantage touchées par la plaie du travail le dimanche à cause de l'implantation de

45 Omer Héroux, Le Devoir, 26 mars 1936.

46 Charles-Edouard Dorion, "La loi du repos", La Vie Nouvelle, 9 (février 1926) : 72. 
l'industrie de la pulpe sur leur territoire, les grandes villes, par d'autres industries, commencent à souffrir du même fléau. Certains entrepreneurs de construction en prennent à leur aise avec le précepte dominical. Dans les domaines du commerce et de l'alimentation des abus sont signalés. Devant la gravité du mal, l'Episcopat du Québec désigne le mois d'avril 1929 comme "le mois du dimanche". La presse catholique reproche au gouvernement d'appliquer rigoureusement la loi des alcools mais d'agir avec beaucoup plus de mollesse envers les violateurs du dimanche.

Relativement à l'ensemble du Canada, c'est au Québec que le repos dominical est le plus difficilement observé. Même si cela est vrai, le clergé envie peu la stricte observance que l'on reconnaît ailleurs. "Sans doute, il y a deux manières de comprendre cette observation: la manière protestante et la manière catholique. La nôtre paraît plus large, plus humaine, plus conforme à une religion d'amour. Nous n'avons pas l'intention de lui substituer celle de Toronto." 47 Mais les dirigeants et les associations catholiques n'en continuent pas moins de réclamer une meilleure observance de la loi du dimanche. Comment expliquer alors la pauvre réputation du Québec quant au respect du précepte dominical ? Cela tient à deux causes différentes: en premier lieu, à la nature de l'industrie en activité au Québec; en second lieu, aux forces occultes qui agissent au sein du gouvernement. La pulpe est la principale industrie. Or, il n'est pas facile d'arrêter le flottage du bois le dimanche ou d'empêcher l'entretien des moulins. En vertu d'un amendement inclus dans la loi en 1925, les Juifs ont le privilège de travailler le dimanche s'ils ont observé le sabbat. Or, un bon nombre de ces citoyens s'adonnent au commerce aussi bien le samedi que le dimanche. En posant ce geste, ils s'attirent la réprobation des milieux catholiques et soulèvent l'animosité de leurs concurrents canadiens-français.

Il est sans doute difficile au gouvernement d'exiger la cessation de tout travail dominical dans la grande industrie. Cependant, les compagnies de pâtes et papiers jouissent d'une indulgence que les partisans de l'observance du précepte dominical jugent suspecte et qu'ils n'expliquent que par des accointances cachées. Par ailleurs, l'élément juif, depuis qu'il a acquis une importance numérique assez considérable, a toujours accordé sa confiance au parti libéral. Le groupe ministériel compte dans ses rangs les deux seuls représentants de la minorité juive à l'Assemblée législative: Joseph Cohen, député de Montréal-Saint-

47 Joseph-Papin Archambault, Le Devoir, 3 mai 1933. 
Laurent et Peter Bercovitch, député de Montréal-Saint-Louis. Leur présence peut être l'une des raisons du peu de zèle manifesté par le premier ministre pour l'abolition du privilège juif de travailler le dimanche. Devant l'insistance de la presse catholique, le chef du gouvernement soumet à la Cour d'Appel "la question de savoir si la législature provinciale a le droit d'abroger l'article 7 de la loi provinciale du dimanche ..." 48 A l'unanimité, le plus haut tribunal du Québec reconnaît au gouvernement le pouvoir d'abolir le privilège juif de travailler le dimanche, par une décision rendue le 3 décembre 1935. Le discours du Trône du 24 mars 1936 annonce la suppression du privilège juif:

Le gouvernement vous priera également, afin d'assurer le respect du dimanche, qui est la base des sociétés véritablement chrétiennes, et à la suite d'un jugement de la Cour d'Appel, d'annuler toute exception qui existe dans nos lois en faveur d'une classe de nos concitoyens. ${ }^{49}$

L'organe officiel de l'Archevêché de Montréal prend acte des derniers gestes du gouvernement et conclut "que le success couronne habituellement les luttes vigoureuses, méthodiques, tenaces". ${ }^{50}$ Une observation attentive des événements permet d'affirmer que l'abolition du privilège juif est loin d'avoir guéri la plaie du travail le dimanche. ${ }^{51}$

\section{Le suffrage féminin}

La plupart des problèmes soulevés illustrent la tension qui a marqué les relations entre l'Eglise et l'Etat sous Louis-Alexandre Taschereau. S'occuper de législation sociale sans susciter les appréhensions des milieux ecclésiastiques est un défi presque impossible à relever à cette époque. L'analyse des événements indique que l'Eglise catholique, en matière de lois sociales, accepte facilement le maintien du statu quo, mais repousse avec la dernière énergie toute volonté d'innovation. C'est l'évidence même qu'il n'y a point d'innovation sans risques. Cet immobilisme est fort bien caractérisé par la question du suffrage féminin. ${ }^{52}$

48 Le Patriote, 6 juin 1935.

49 Cité par Omer Héroux, Le Devoir, 26 mars 1936.

50 La Ligue du Dimanche, "L'abrogation du privilège juif", La Semaine religieuse de Montréal, 95 (juin 1936) : 400.

51 Le lecteur pourra consulter le RAPPORT de la Commission d'enquête sur l'observance du dimanche dans les usines de pâtes et papiers du Québeo (Québec, 1966).

52 Il serait intéressant de consulter les Etudes préparées pour la Commission royale d'enquête sur la situation de la femme au Canada, en particulier l'étude de Micheline Johnson, intitulée "Histoire de la condition de la femme dans la province de Québec" et publiée dans l'annexe 8 (1971). 
C'est d'ailleurs l'un des rares moments où les vues des autorités civiles et religieuses ont été identiques. Puisant à une même philosophie conservatrice, les deux pouvoirs s'entendent pour garder l'élément féminin de la population québécoise en dehors des contestations politiques. A cette raison principale, s'ajoutent d'autres motifs. Le pouvoir religieux s'oppose au suffrage féminin craignant l'émancipation trop rapide de la femme et l'attiédissement de la foi dans les familles; le pouvoir civil redoute d'accorder le droit de vote aux femmes car il présume que ces nouvelles voix iraient à l'adversaire, le parti conservateur recrutant de nombreux alliés parmi les membres du clergé et la femme canadienne-française étant facilement perméable à l'influence religieuse. Si, aux élections fédérales, l'Eglise catholique accepte le suffrage féminin, elle s'en justifie du fait que, face à une majorité anglo-protestante, elle doit pouvoir compter sur le plus grand nombre possible d'électeurs de foi catholique afin d'obtenir une législation plus conforme à sa doctrine.

$\mathrm{Au}$ Canada, les femmes et les parentes des soldats déjà sous les armes ont pu voter aux élections de 1917. Aux élections de 1921, toute la population féminine du Canada peut exercer son droit de vote. Ainsi, au Québec, les femmes contribuent à donner un mandat unanime au parti libéral. Ce sont des députés libéraux qui occupent les soixante-cinq sièges accordés au Québec, aux élections fédérales de 1921. Presque aussitôt, les gouvernements provinciaux imitent le gouvernement central et accordent le droit de vote aux femmes.

Devant la vague féministe qui semble balayer le Canada, les autorités religieuses du Québec s'alarment. Dès 1920, le cardinal Bégin, archevêque de Québec, publie une lettre pastorale sur les conditions religieuses de la société canadienne. Il range le féminisme au nombre des périls qui menacent la famille:

Cette fausse conception du mariage n'est pas sans relations avec les doctrines féministes qui tendent à détourner la femme de ses devoirs d'épouse et de mère pour la jeter sur la place publique. ${ }^{53}$

Poursuivant son offensive, le 11 janvier 1922, l'Episcopat du Québec adresse une lettre confidentielle au premier ministre Louis-Alexandre Taschereau pour lui faire connaître son opposition formelle au suffrage féminin:

Le premier de ces vœux, c'est que la Législature de notre province s'abstienne d'accorder aux femmes le droit de suffrage politique.

${ }^{53}$ L.-N. Bégin, "Lettre pastorale", La Semaine religieuse de Québec, 32 (juillet 1920): 741. 
Dès 1909, les Pères du Premier Concile national canadien condamnaient unanimement la tendance moderne par laquelle on veut établir entre l'homme et la femme "une similitude de droits" et faire entrer la femme "dans une ridicule et odieuse rivalité avec l'homme"; et les Pères ajoutaient: "La mise en pratique de pareilles théories serait funeste à la femme et à la famille, et amènerait à bref délai la déchéance de l'une et la ruine de l'autre."

Ces paroles nous semblent atteindre directement le suffrage politique féminin; et la Législature de Québec répondrait à notre vif désir en rejetant, comme elle l'a fait judicieusement dans le passé, toute proposition de loi destinée à créer, pour les femmes le droit de vote dans les élections politiques de notre province. ${ }^{54}$

Sur cette question, l'appui du pouvoir politique sera indéfectible. Le gouvernement du Québec n'a aucunement l'intention de favoriser les féministes et son chef le fait savoir clairement à tous les archevêques et évêques du Québec:

Personnellement, je suis opposé au suffrage féminin. Plusieurs de mes collègues partagent cette manière de voir; d'autres y sont favorables. La députation est divisée à ce sujet, et si j'ai bien compris les opinions exprimées dans les journaux, les théologiens eux-mêmes sont loin d'être d'accord.

Le problème, qui en est plutôt un de morale et d'ordre social, n'est donc pas facile à résoudre. ${ }^{5 \bar{b}}$

Pour faire échec au projet de loi visant à obtenir le suffrage féminin aux élections provinciales, l'administrateur du diocèse de Québec, Mgr Paul-Eugène Roy, pendant la session de 1922, exprime son opposition irréductible à l'émancipation féminine dans une lettre aux Membres du Comité de Propagande contre le suffrage féminin:

Permettez-moi de vous féliciter de cette heureuse inspiration qui répond parfaitement aux vues de tout l'épiscopat de la province et qui fait grand honneur à votre sens social et à votre esprit chrétien.

Une législation qui ouvrirait la porte au suffrage des femmes serait un attentat contre les traditions fondamentales de notre race et de notre foi, et les législateurs qui mettraient la main à une telle législation commettraient une grave erreur sociale et politique. ${ }^{56}$

54 ANQ, Fonds Louis-Alexandre Taschereau, Lettre des archevêques et évêques du Québec à Louis-Alexandre Tachereau, 11 janvier 1922.

55 ANQ, Fonds Louis-Alexandre Taschereau, Lettre de Louis-Alexandre Taschereau à Mgr Paul-Eugène Roy, 20 janvier 1922.

56 P.-E. Roy, "Lettre de Mgr l'Administrateur", La Semaine religieuse de Québec, 34 (23 février 1922) : 408. 
La lettre de Mgr Paul-Eugène Roy contre le suffrage féminin reçoit sa consécration, le 19 mars 1922 , lorsque le cardinal Bégin, du Collège Canadien de Rome où il séjourne, fait connaître son acquiescement en ces termes:

L'entrée des femmes dans la politique, même par le seul suffrage, serait, pour notre province, un malheur. Rien ne la justifie, ni le droit naturel, ni l'intérêt social. Les autorités romaines approuvent nos vues qui sont celles de tout notre épiscopat.

Nos professeurs, dans leur enseignement, devront tenir compte de cette direction. ${ }^{57}$

Quant au gouvernement, il éconduit avec urbanité une délégation de deux cents femmes venues au Parlement soumettre le point de vue du mouvement féministe:

Dans aucun pays français ou latin, on n'a accordé le droit de suffrage aux femmes. Je me demande ce que pourra gagner la femme en ayant le droit de vote. Sa place n'est pas dans la politique, c'est au foyer, dans la famille où elle peut exercer la meilleure influence. En se mêlant aux luttes politiques, la femme perdra peut-être le respect qu'on lui doit. ${ }^{58}$

Le projet de loi présenté par le député Henry Miles n'a même pas fait l'objet d'un débat.

Il faut attendre l'année 1927 pour revoir une autre délégation au Parlement. En même temps, un autre projet de loi est présenté à l'Assemblée législative en faveur du suffrage féminin. Il est "défait à une grosse majorité". ${ }^{59}$ Les partisans du suffrage féminin n'affichent aucun masque funéraire après la défaite. "Le spectacle assez rare de voir le premier ministre et le chef de l'opposition votant ensemble contre l'ennemi (?)" 60 les porte plutôt à railler qu'à rager. Ils constatent, à l'étude des arguments défavorables au suffrage féminin, que "c'est un devoir sacré pour la femme de voter aux élections fédérales, mais c'est la faire déchoir que de lui donner ce même droit aux élections provinciales". ${ }^{61}$ D'autant plus que le Québec est le seul endroit de l'Amérique du Nord où l'on refuse aux femmes le droit de citoyenneté.

57 Louis-Nazaire Bégin, "Au sujet du vote des femmes", La Semaine religieuse de Québec, 34 (avril 1922) : 536.

58 L.-A. Taschereau, "Les femmes ne doivent pas se mêler de politique", L'Enseignement primaire, 43 (mars 1922) : 456 .

${ }^{59}$ Le Canada, 12 mars 1927.

60 Socius, Le Monde Ouvrier, 19 mars 1927.

61 Ibid. 
Par la suite, vers la mi-carême, les femmes favorables au suffrage féminin accomplissent leur pèlerinage annuel au Parlement de Québec. Les députés les accueillent avec joie car elles jettent une note de fantaisie dans l'auguste enceinte parlementaire. Mais les élus du peuple n'en continuent pas moins, année après année, à dénier aux femmes le droit de voter aux élections provinciales. Chaque nouvelle tentative est repoussée avec la même constance. A la veille des élections provinciales du 25 novembre 1935, l'Alliance Canadienne pour le vote des femmes $d u$ Québec sert une mise en demeure au premier ministre du Québec. La lettre, signée par la présidente, mademoiselle Idola Saint-Jean, et quelques femmes, a été adressée au bureau du premier ministre à Québec par un membre de l'organisation libérale de Montréal qui n'avait pu la lui remettre à l'occasion de l'assemblée politique du marché Saint-Jacques. Le texte est bref mais très ferme:

Nous venons vous prier de nous déclarer au cours de cette campagne si vous êtes favorable au vote féminin et si, étant élu, vous exercerez votre influence pour obtenir pour les femmes de cette province un statut politique égal à celui de leurs sœurs de toutes les autres provinces du Canada. ${ }^{62}$

La demande n'a pas reçu la faveur du chef du gouvernement puisque l'Assemblée législative refuse encore, le 27 mai 1936, d'accorder le droit de vote aux femmes. Ce refus n'est pas unanime. Cependant, une majorité des représentants de tous les partis demeure irréductible et, chaque fois, le projet de loi est repoussé avec la même ténacité. L'élément masculin de la population manif $\epsilon$ ste peu de sympathie envers la cause féministe, mais l'obstacle le plus considérable vient des femmes elles-mêmes qui, en grand nombre, rejettent le droit que réclament quelquesunes de leurs semblables. L'attitude de la majorité féminine s'explique plus facilement quand on sait que Marie Gérin-Lajoie, première présidente du Comité provincial du suffrage féminin, est obligée de démissionner, en novembre 1922, sous la pression de l'Eglise catholique. ${ }^{63} \mathrm{La}$ question du suffrage féminin prend une importance particulière en ce qu'elle révèle bien la mentalité de l'époque. Plusieurs députés de l'Assemblée législative et plusieurs membres de la hiérarchie catholique et du clergé, indépendamment de leur assujettissement politique ou religieux, sont peu favorables au suffrage féminin parce qu'ils ont fréquenté

62 ANQ, Fonds Louis-Alexandre Taschereau, Lettre de Idola SaintJean et al. à Louis-Alexandre Taschereau, 18 novembre 1935.

03 Catherine Lyle Cleverdon, The Women Suffrage Movement in Canada (Toronto, 1950), 230. 
les mêmes collèges et ont reçu le même enseignement philosophique. Si le gouvernement québécois est réactionnaire relativement aux autres gouvernements provinciaux, il faut reconnaître que l'opposition de l'Eglise catholique du Québec ne reflète pas nécessairement la pensée de Rome.

\section{L'éducation}

Sous le gouvernement Taschereau, le monde de l'éducation fait souvent les manchettes des journaux. Cette affirmation n'indique pas nécessairement que le développement de l'instruction, au Québec, de 1920 à 1936, a été continu et linéaire. Elle signifie que, si des mesures progressives ont été proposées, en d'autres occasions, des rumeurs de "contrainte scolaire" et de ministère de l'Instruction publique ont ameuté les principaux organes de la presse catholique.

La question de l'instruction obligatoire est un sujet de brûlante actualité pendant les seize années du mandat du premier ministre Louis-Alexandre Taschereau. La lutte entre les partisans et les adversaires de l'instruction obligatoire est empreinte d'une vive animosité. Touchant la campagne en faveur de l'instruction obligatoire, de nombreuses requêtes avaient été signées pour s'y opposer. A propos de l'une d'elles, l'auteur d'un ouvrage favorable à l'obligation scolaire rapporte le fait bizarre suivant:

A petition was presented to the Assembly by the Deputy for Charlevoix-Saguenay. This petition is so ridiculous that it deserves more than a word of exposure. It was signed by more than a hundred names, at the head being the worthy priest for St. Hilarion. On the first page there were about 30 signatures, and half of these men so illeterate they could not even sign their names but had to make their mark. ${ }^{64}$

Pour sa part, l'inspecteur général C.-J. Magnan exprime l'opinion suivante:

1. Le progrès toujours désirable en matière d'instruction publique est possible chez nous sans le recours à la contrainte;

2. L'instruction obligatoire qui a fait faillite partout est sûrement un instrument de déchristianisation. ${ }^{65}$

64 I.O. Vincent, The Right Track (Toronto, 1920), 183.

65 Cité par l'Echo de Saint-Justin, $1^{\text {er }}$ juillet 1922. 
En plus des individus, les journaux participent avec frénésie à la querelle de l'instruction obligatoire. Certains font connaître leur opinion de façon fort peu révérencieuse:

L'Action catholique se glorifie de ce que le Québec est le dernier rempart de l'ignorance, c'est la fierté du coq qui chante sur un tas de fumier. Il y a là vraiment de quoi se réjouir d'être à la queue de la civilisation. Où d'autres pleureraient sur un état de chose aussi lamentable, elle se bat les flancs pour s'arracher queiques cris rauques qui sont sa manière de témoigner sa joie.68

Si l'on fait le bilan de la période de 1920 à 1936, on ne peut soutenir que l'éducation a accompli des bonds prodigieux et que le régime politique a favorisé une évolution rapide. C'est au début de l'administration de L.-A. Taschereau que les mesures les plus audacieuses ont été votées. Dès la session de 1921, le gouvernement propose une "loi autorisant certains octrois pour fins d'éducation et certains emprunts nécessaires à ces fins". ${ }^{67}$ Par cette mesure, l'Université Laval, l'Université de Montréal et l'Université McGill reçoivent la somme d'un million de dollars. La première session de 1922 est riche en mesures législatives touchant le domaine de l'enseignement. L'Assemblée législative vote une loi autorisant une subvention de $\$ 100,000$ au Bishop's College. Ce montant est payable par versements annuels de $\$ 20,000$ chacun. A leur tour, les collèges classiques du Québec reçoivent une somme annuelle de $\$ 230,000$, soit $\$ 10,000$ pour chacun d'eux. Ces maisons d'enseignement pourront consacrer l'argent à l'achat de livres, à l'établissement de laboratoires de sciences et au perfectionnement de l'enseignement secondaire. Les écoles protestantes ne sont pas oubliées et reçoivent annuellement $\$ 40,000$ à compter de $1922 .{ }^{.8}$

L'établissement des Ecoles techniques et de l'Ecole des Hautes Etudes commerciales n'avait pas reçu l'assentiment des autorités religieuses. La loi de 1922 "créant les écoles des beaux-arts de Québec et de Montréal" ${ }^{69}$ est aussi mal accueillie. Toutes ces institutions échappent à la juridiction de l'Eglise catholique et relèvent directement de l'Etat. Depuis mai 1915, par suite de son affiliation à l'Université Laval, l'Ecole des Hautes Etudes commerciales n'entre plus dans cette catégorie. La même année 1922 voit le gouvernement, par l'entremise de Athanase David, proposer une loi "pour encourager la production d'œuvres litté-

66 Philippe EGALITE, Le Peuple, 30 septembre 1922.

67 Statuts de Québec, 11 George V (1921), chap. 3: 39.

68 Statuts de Québec, 12 George V (1922), chap. 4 et 5: 27.

69 Ibid.: 218. 
raires ou scientifiques". ${ }^{70}$ Un prix de $\$ 5,000$ est accordé à la réalisation de travaux littéraires ou scientifiques.

Ces gestes du gouvernement marquent tout au moins un désir de promouvoir l'éducation au Québec. Mais peut-on parler d'une volonté bien arrêtée de donner au peuple québécois les outils nécessaires à son épanouissement intellectuel? L'étude des différentes lois votées par le gouvernement Taschereau après 1922 n'apporte pas une réponse affirmative à la question. On peut proposer quelques explications. D'une part, les intérêts financiers n'ont aucune raison d'appuyer le gouvernement dans une politique de libéralisation du savoir. Les firmes étrangères viennent au Québec parce qu'elles y trouvent des ressources et une main-d'œuvre docile et elles n'ont pas pour but de combattre l'analphabétisme. D'autre part, l'Eglise catholique s'oppose opiniâtrement aux initiatives gouvernementales dans le domaine de l'instruction publique. Il n'est que de songer à la loi de 1907 établissant des écoles techniques à Montréal et à Québec et créant l'Ecole des Hautes Etudes commerciales, sous le gouvernement de Lomer Gouin. L'opposition du pouvoir religieux face à l'intervention de l'Etat va se poursuivre sous le gouvernement Taschereau. Ainsi, le projet de loi de 1926 destiné à centraliser entre les mains d'une "Corporation des écoles techniques" ${ }^{71}$ l'administration de l'Ecole des Hautes Etudes commerciales (1911), des trois écoles techniques (1907) de Montréal, de Québec et de Hull et des deux écoles des beaux-arts (1922) de Montréal et de Québec suscite les alarmes d'ecclésiastiques intransigeants et de laïques réactionnaires. Même l'organe officiel de l'archevêché de Québec manifeste ouvertement sa désapprobation:

\section{(...)}

En effet, une fois l'enseignement d'Etat consacré par la législation, ne fût-ce que pour quelques rares écoles techniques ou autres, la brèche est faite et par cette brèche des hommes publics moins bien intentionnés que ceux qui nous gouvernent aujourd'hui pourraient plus facilement faire entrer, un jour, l'école populaire neutre.

A ce sujet, il est donc permis de regretter l'existence chez nous d'écoles gouvernementales qui ouvrent chaque année la voie de plus en plus large à l'Etat pédagogue. La solution du problème, ne serait-ce pas l'affiliation de toutes les écoles créées par l'Etat à nos différentes universités, qui ont pour mission de donner un enseignement confessionnel?

${ }^{70}$ Ibid.: 221.

71 Statuts de Québec, 16 George V (1926), chap. 49: 273. 
Nous avons confiance que nos législateurs, tous soucieux de respecter nos traditions, ne manqueront pas de prendre en bonne part ces observations, uniquement inspirées par le souci de voir nos meilleurs intérêts sauvegardés de la façon la plus efficace. ${ }^{72}$

L'Eglise catholique contrôlait l'enseignement primaire, secondaire et supérieur et elle refusait que l'enseignement "professionnel" lui échappe.

La presse dévouée au gouvernement se hâte de rassurer les plus angoissés:

On a voulu en certains milieux insinuer que le gouvernement conduisait la province vers l'école neutre tout simplement, parce qu'il avait le contrôle de certaines écoles.

Mais comme le démontre le premier ministre, le gouvernement ne garde le contrôle que des écoles qui peuvent tomber sous sa juridiction telles que l'Ecole des Hautes Etudes Commerciales, l'Ecole technique, les écoles des Beaux-Arts.

Ce sont là des écoles où les enfants de toutes les dénominations religieuses, appartenant à toutes les races sont admis: peut-on raisonnablement placer ces écoles sous le contrôle exclusif du clergé catholique. ${ }^{73}$

La moindre allusion à un éventuel ministère de l'Instruction publique lance aussitôt les organes de la presse catholique sur le sentier de la guerre. Quant à la presse neutre, elle ne peut comprendre qu'un principe aussi sage que le droit à l'éducation ne puisse s'appliquer au Québec. Tout être humain a droit au savoir comme à l'air, à l'eau et au soleil. Il est à espérer que la masse ouvrière obligera le gouvernement à imposer l'instruction obligatoire puisque l'élite faillit à son devoir. ${ }^{74}$

L'éditorialiste du journal ouvrier, Julien Saint-Michel, exprime une vérité fort juste. Si l'Eglise catholique s'oppose à "la contrainte scolaire", l'élite politique manifeste peu d'enthousiasme en faveur de l'instruction obligatoire. Dans toutes ses déclarations officielles, le premier ministre du Québec proteste de son désir de respecter la volonté des autorités religieuses et de ne pas intervenir dans le domaine de l'enseignement:

72 La Direction, "Les Ecoles d'Etat", La Semaine religieuse de Québec, 39 (mars 1926) : 466 .

73 Le Canada, 26 avril 1926.

74 Julien Saint-Michel, Le Monde Ouvrier, 10 août 1929. 
Nous n'avons pas de ministère de l'Instruction publique dans notre province. Des associations ouvrières nous l'ont demandé maintes fois, des journaux l'ont aussi réclamé. Avec la grande majorité de mon parti, j'y suis hostile, parce que nous avons quelque chose de mieux et de plus stable.

Notre conseil de l'Instruction publique composé de tous les évêques de la province et d'un nombre égal de laïques dévoués et instruits offre, il me semble, des garanties qu'on pourrait peut-être nous envier ailleurs. Les ministères et les ministres passent et changent, mais ce conseil où siègent des éducateurs avertis, hors des influences politiques, offre un caractère de permanence et de direction saine auxquelles je craindrais de substituer les nôtres.

Le Conseil de l'Instruction a le contrôle absolu de l'instruction élémentaire dans notre province. ${ }^{75}$

(...)

Il paraît juste d'affirmer que Louis-Alexandre Taschereau, l'ensemble de ses ministres et un bon nombre de députés entretiennent sur la question de l'obligation scolaire les mêmes sentiments que la hiérarchie catholique. Pour eux, le savoir est le privilège d'une minorité et il ne saurait être question de rendre obligatoire la fréquentation des écoles. ${ }^{76}$

\section{Les pensions de sécurité de la vieillesse}

Avec l'abolition du privilège juif relativement au travail du dimanche, la loi des pensions de vieillesse est le chant du cygne du gouvernement Taschereau. Pendant près de dix ans, le Québec a refusé d'appliquer la loi fédérale, sanctionnée par le Parlement

${ }^{75}$ Cité par Le Monde Ouvrier, 1er mai 1926.

76 L'ouvrage suivant est à consulter: Victrice Lessard, L'Instruction obligatoire dans la province de Québec. Thèse de $\mathrm{Ph}$. D. en éducation (Université d'Ottawa, 1962). C'est en quelque sorte une vue panoramique de la question. Nous différons d'avis, cependant, lorsque l'auteur énonce l'opinion suivante: "Enfin, dans un contexte plutôt politique, on pourrait tenter une étude sur l'action du parti libéral de l'origine du mouvement à la législation f'nale de 1943. De l'honorable Honoré Mercier, en effet, jusqu'à l'honorable Adélard Godbout, tous les premiers ministres libéraux, à l'exception de l'honorable Alexandre Taschereau peut-être, ont agréé ou favorisé l'instruction obligatoire au grand dam du parti d'opposition." Nous ne voyons pas en quoi S.-N. Parent et Lomer Gouin ont prôné l'instruction obligatoire. L'établissement de l'Ecole des Hautes Etudes commerciales et d'écoles techniques n'est pas suffisant, à notre avis, pour affirmer que Lomer Gouin a favorisé le principe de l'instruction obligatoire.

Louis-Philippe Audet a écrit un article sur le sujet. Voir Les Cahiers des Dix (Montréal, 1959), XXIV: 133 à 150. Il est à remarquer que l'auteur passe sous silence presque complètement la période de Louis-Alexandre Taschereau. 
du Canada, le 31 mars 1927. ${ }^{77}$ Elle autorise le versement d'une somme annuelle de $\$ 240$. à tous les vieillards âgés de plus de 70 ans. Le gouvernement fédéral s'engage à rembourser $50 \%$ des montants versés par les provinces au titre d'assistance aux vieillards. En 1931, la contribution fédérale sera portée à $75 \%$.

Divers motifs sont mis de l'avant pour expliquer le refus de Louis-Alexandre Taschereau. Ce dernier, à l'instar de ses prédécesseurs, adopte une attitude nettement autonomiste. Chaque session, chaque discours est une occasion pour le chef du gouvernement de réitérer sa volonté d'être maître chez lui. A la session de 1926, il impose une politique d'embargo des ressources hydro-électriques vers les Etats-Unis, mais non vers les autres provinces. La prohibition de l'exportation de l'énergie hydroélectrique force les Américains à venir installer leurs usines au Québec. De la même façon, le premier ministre du Québec s'opposera à la canalisation du fleuve Saint-Laurent. Par une résolution votée à l'Assemblée législative ${ }^{78}$, il avertit le gouvernement fédéral que pas une goutte d'eau ne doit être enlevée au Québec. Aussi, faut-il être peu surpris de constater son opposition à l'établissement, par le gouvernement fédéral, d'un régime d'assistance aux vieillards.

Certains discernent dans l'attitude du premier ministre du Québec, une réaction de caractère atavique qui l'incite à repousser toute aide étrangère. Depuis longtemps au Québec, l'Eglise catholique enseigne à ses fidèles qu'ils doivent veiller au bonheur de leurs vieux parents. Elle leur fait un devoir d'assurer leur subsistance et de faire en sorte que le crépuscule de leur existence s'écoule sans heurt et sans problème jusqu'au moment du repos éternel. Cette tradition, fortement ancrée dans la population québécoise, explique en partie la répugnance que manifeste le gouvernement envers la loi des pensions de vieillesse. ${ }^{79}$

Ce n'est qu'en 1936, sous la pression de plus en plus forte de contribuables québécois ${ }^{80}$ et sous le poids d'une certaine lassitude, que le chef du gouvernement du Québec accepte de par-

77 Statuts du Canada, 17 George V (1926-1927), chap. 35: 167.

78 Cette résolution présentée par Athanase David est adoptée en troisième lecture le 14 janvier 1932 .

79 Gérald Godin laisse entendre que Louis-Alexandre Taschereau s'est opposé à la loi des pensions de vieillesse parce qu'il siégeait au conseil d'administration de certaines compagnies d'assurance. Québec-Presse, 19 mars 1972.

${ }^{80} \mathrm{La}$ correspondance de L.-A. Taschereau des années 1935 et 1936 contient un nombre considérable de lettres dont les auteurs interrogent le premier ministre au sujet des pensions de vieillesse. 
ticiper au plan fédéral de pension de sécurité de la vieillesse. La loi reçoit la sanction royale le 10 juin ${ }^{81}$ et elle est mise en vigueur le $1^{\text {er }}$ août de la même année. Le 11 juin 1936, LouisAlexandre Taschereau remet sa démission et annonce la dissolution de la $19^{\circ}$ législature.

\section{Conclusion}

Plusieurs questions qui relèvent de la législation sociale n'ont pas fait l'objet d'une étude circonstanciée. En plus des raisons déjà données, on peut ajouter que le cadre de cet article n'aurait pas permis de les commenter à fond. Qu'il suffise de mentionner l'affaire des écoles juives ${ }^{82}$, la colonisation, l'heure avancée, l'hygiène publique, le syndicalisme catholique et la législation du travail.

Il n'est pas facile de résumer l'œuvre sociale du gouvernement Taschereau. Pour certains, les progrès qui ont été accomplis au Québec, de 1920 à 1936, sont davantage le résultat d'une longue possession du pouvoir que le fruit d'une politique avantgardiste et progressive. Sans vouloir trancher le débat, on peut affirmer que des mesures audacieuses ont vu le jour au cours de cette période.

Plusieurs n'ont retenu de ces années de l'histoire du Québec qu'une image de corruption et de népotisme. Sans doute, peut-on reprocher à Louis-Alexandre Taschereau son manque de surveillance et de contrôle dans les dépenses des deniers publics. Il siégeait lui-même au conseil d'administration de nombreuses compagnies et ne s'en formalisait pas: "Au contact de tous ces hommes d'affaires, j'acquiers une expérience précieuse dont la province et toute la population bénéficient." 83 Il n'en demeure pas moins que des conflits pouvaient naître entre le bien de la province et les intérêts de sociétés particulières.

On ne peut parler de législation sociale sans traiter des relations entre l'Eglise et l'Etat. Au Québec, le rôle de l'Eglise a toujours été prépondérant. Encore au début du $\mathrm{XX}^{\mathrm{e}}$ siècle, les chefs politiques prenaient souvent l'avis des autorités religieuses

${ }^{81}$ Statuts de Québec, 1 Edouard VIII (1936), chap. 1: 3.

$82 \mathrm{~A}$ ce sujet, le lecteur pourra se reporter à l'article de Robert Rumilly publié dans $\mathrm{RHAF}, \mathrm{X}, \mathrm{n}^{\circ} 2$ (Montréal, 1956) : 222 à 224. On pourra également consulter la thèse de Harold Ross, The Jew in the Educational System of the Province of Quebec (Thesis presented to McGill University, Montreal 1947).

83 Le Canada, 2 novembre 1935. 
avant de poser un geste de quelque portée même lorsque l'incidence religieuse était à peine perceptible. On croit déceler dans quelques-uns des gestes de L.-A. Taschereau une certaine impatience devant le conservatisme de l'Eglise catholique. En revanche, le premier ministre du Québec était heureux de s'appuyer sur l'autorité religieuse pour légitimer des prises de positions nettement réactionnaires. Dans l'ensemble, on peut conclure à un début d'émancipation du gouvernement envers une puissance qui exerçait une influence sans lien vraiment logique avec sa mission spirituelle. 